\title{
Haptic/VR Assessment Tool for Fine Motor Control
}

\author{
Christophe Emery $^{1}$, Evren Samur ${ }^{1}$, Olivier Lambercy ${ }^{2}$, \\ Hannes Bleuler ${ }^{1}$ and Roger Gassert ${ }^{2}$ \\ 1 Ecole Polytechnique Fédérale de Lausanne, Robotic Systems Lab, Switzerland \\ 2 ETH Zurich, Rehabilitation Engineering Lab, Switzerland \\ \{christophe.emery, evren.samur, hannes.bleuler\}@epfl.ch, \\ \{olambercy, gassertr\}@ethz.ch
}

\begin{abstract}
The Nine Hole Peg Test (NHPT) is routinely used in clinical environments to evaluate a patient's fine hand control. A physician measures the total time required to insert nine pegs into nine holes and obtains information on the dexterity of the patient. Even though this method is simple and known to be reliable, using a virtual environment with haptic feedback instead of the classical device could give a more complete diagnosis which would isolate different constituting components of a pathology and objectively assess motor ability. Haptic devices enable extracting a large quantity of information by recording the position and the exerted forces at high frequency $(1 \mathrm{kHz})$. In addition to the creation of a realistic virtual counterpart of the NHPT, the present work also includes the implementation of real-time data analysis in order to extract meaningful and objective scores for the physician and the patient. A healthy group of volunteers performed the real and virtual tests which yielded a baseline for the scores of the different measured mobility parameters. Once calibrated, the virtual test successfully discriminates different mobility dysfunctions simulated by a healthy subject.
\end{abstract}

Keywords: haptics, virtual reality, clinical, assessment, dexterity.

\section{Introduction}

Robotics is widely used in industry because of its ability to precisely and repetitively control positions and interaction forces, and provide objective measures of various parameters, which has not only motivated its use in physical therapy, but also in clinical diagnostics. A haptic rendering system consisting of a screen (for visual feedback) and a haptic interface (for force feedback) allows immersing the user into a virtual scene [5] and tracking the movements of the user at a high frequency. A growing number of studies demonstrate that these systems give quite reliable information on the user's dexterity, for instance precision and stability [3]. Nevertheless, there are major issues that can interfere with these measures, such as the quality of the visual or force feedback, the limited workspace or the user's inability to link the scene on the screen to a displacement of the interface. However, this field is progressing quickly and these issues tend to disappear as the realism increases and the technologies evolve. Using a haptic rendering system to provide a recognized, objective measure of the dexterity of a human could soon be a reality. 

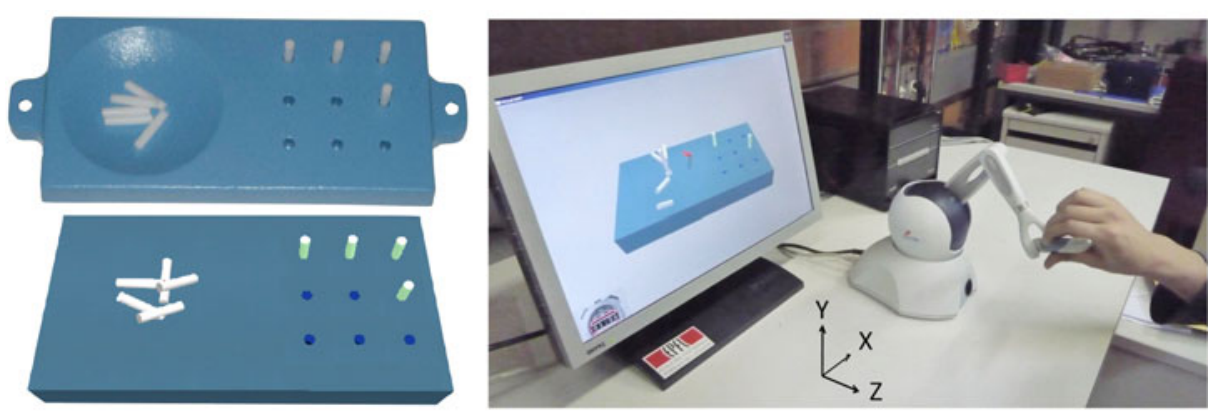

Fig. 1. Left: Conventional Nine Hole Peg Test (top) and its virtual counterpart (bottom). Right: Virtual NHPT setup.

The Nine Hole Peg Test (NHPT) is a common clinical tool to assess the dexterity of a subject with impaired mobility, and consists of the insertion and removal of nine pegs in nine holes (Fig. 1) with one hand as fast as possible. The total time is measured and allows the physician to evaluate the dexterity of the patient. However, this measure gives no information on specific parameters such as reaction time, stability or speed. Knowing that an insertion task requires a wide range of abilities such as upper limb mobility, movement coordination, precision and stability, a test that could extract more parameters than only the total execution time, but with the simplicity of the NHPT, would be of great benefit to obtain a better assessment of dexterity.

The goal of this study is to develop an experimental setup that takes advantage of the classical NHPT (easy to administer, standardized, established) and of a virtual environment (controlled environment, adjustable parameters, quantitative and objective measures), while trying to reduce the drawbacks of the haptic rendering system (vibrations, contact instabilities and realism) [2]9]. Previous studies suggested the potential of combining the NHPT with haptics technology [1[10]. However, these studies did not extract parameters related to orientation (important during grasping and insertion of pegs) or simulate active grasping of the pegs (e.g. over an integrated switch). Here, we propose a novel method to determine dexterity parameters based on the measurements extracted from the haptic device in order to provide objective information in an intuitive manner to physicians and patients.

\section{Materials and Methods}

\subsection{Nine Hole Peg Test}

The apparatus used in this study is the Rolyan(c) 9-Hole Peg Test consisting of a blue plastic board with nine holes on one side and a round container on the other, and nine white plastic pegs placed in the round container (Fig. 1). The board is $255 \mathrm{~mm}$ long, the pegs are $31.9 \mathrm{~mm}$ long and $6.2 \mathrm{~mm}$ in diameter and the holes are $7.2 \mathrm{~mm}$ in diameter and $12.8 \mathrm{~mm}$ deep. The test is sold for around $80 \$$ and the validity and reliability of this test to evaluate dexterity have been demonstrated by clinical trials [68]. 


\subsection{Virtual NHPT}

The experimental setup consists of a PC (Intel Core 2 Duo with $3 \mathrm{GHz}$ CPU) to run the tests, an LCD screen to display the virtual environment, and a force-feedback device to simulate haptic interactions (Fig. 11). The PHANTOM OMNI $®$ from SensAble Technlogies, Inc., which has 6-DOF positional sensing and 3-DOF force feedback, is used for this study as it has already been applied in similar studies [3/4] and is affordable for clinical use. It's workspace ( $160 \mathrm{~W} \times 120 \mathrm{H}$ x $\left.70 \mathrm{D} \mathrm{mm}^{3}\right)$ is just wide enough to allow a 1:1 scale with the real setup and it's resolution of $55 \mu \mathrm{m}$ is good enough to obtain a fine measure of human movements.

A dual-thread software package is developed to realize the virtual version of the NHPT. The graphic rendering is set up with OpenGL and GLu libraries. The low-level foundational API (hd and hdu) provided by the PHANTOM vendor is used for the interaction with the haptic interface. The haptic and visual loops are updated at a rate of 1000 and $60 \mathrm{~Hz}$, respectively. The coordinate system was set in a way that the X-axis points right and the $\mathrm{Y}$-axis points up.

The objects in the entire virtual scene are modeled as rigid bodies. These include ten cylinders (nine as the pegs (opaque white) and one as the cursor (transparent grey) representing the PHANTOM stylus), nine holes, the board and the ground. The distance between the cursor (or a peg) and the other objects is calculated for collision detection. A linear spring model with a stiffness value of $0.4 \mathrm{~N} / \mathrm{mm}$ is implemented to render the force given by the device when there is a collision. The weight of the pegs is not considered in the simulation. During the peg insertion, the total force corresponds to the vectorial sum of a vertical force due to the penetration into the surface and an inward horizontal force due to penetration into the wall of the hole. This horizontal force is decreased by a factor of 0.15 in order to avoid instabilities (the diameter of the holes is only slightly larger than that of the pegs). Collisions between the pegs are not considered in the simulation. When the user presses one of the two buttons on the stylus, the grip is active and the cursor turns from transparent grey to transparent blue. When the cursor is precisely superimposed with a peg and the grip is active, the cursor becomes opaque red and the peg can then be manipulated. If the button is released, the peg falls from the cursor and its color turns back to opaque white. If the peg is correctly inserted into a hole, it becomes green, the cursor is ejected from the hole and the inserted peg cannot be moved any more.

Subjects and Procedure. Ten healthy volunteers aged between 23 and 38 (mean 26.8 \pm 3.2 ) participated to the evaluation of the virtual NHPT. Two of them were left-handed and eight of them were not familiar with haptic interfaces. The goal of this study was to compare the real and virtual test, and determine baseline parameters to assess dexterity in healthy subjects.

The experiment consisted of two experimental conditions (real and virtual) performed with both hands (dominant and non-dominant). The tasks were crossed between subjects and randomly assigned in order to minimize the effect of learning. A task with the real test consisted of two training sessions followed by three recorded sessions where subjects were told to insert the 9 pegs, one at a time, as fast as possible, only with the tested hand. The test was completed once the nine pegs were inserted into 
the nine holes. Unlike the normal NHPT procedure, subjects were not told to remove the pegs after inserting them. Completion times of the three trials were averaged for the analysis. For the virtual test, the PHANTOM was placed on the side of the tested hand and the subject had to hold the stylus like a pen, with the thumb on the buttons. Raw data including elapsed time $(\mathbf{t})$, positions $(\mathbf{x}, \mathbf{y}, \mathbf{z})$, orientations in polar coordinates $(\phi, \theta)$, forces $\left(F_{x}, F_{y}, F_{z}\right)$ and order of peg manipulation $(\mathbf{P})$, was recorded at $1 \mathrm{kHz}$.

\section{Data Analysis}

The analysis consists of two steps (Fig. 2): first the raw data is divided into sequences using position thresholds [7] to extract raw parameters (velocities, accelerations etc.), then each of these raw parameters is compared to its baseline and contributes to a calculated score that has meaning for both patients and physicians, such as precision, stability or flexibility.

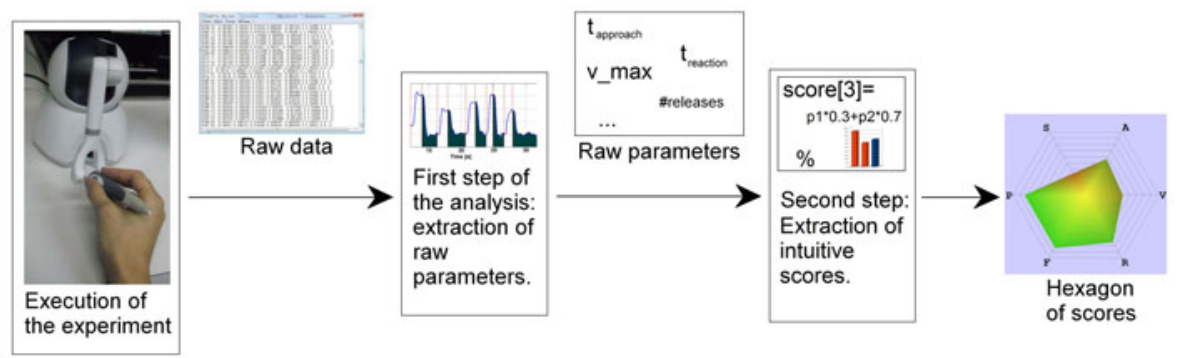

Fig. 2. Schematic representation of the data extraction process

Extraction of the Raw Parameters. For each movement (i.e. movement from a peg to a hole and then to a peg again), we extract nine raw parameters: the reaction time $\left(t_{\text {reac }}\right)$, approach time $\left(t_{\text {appro }}\right)$, mean velocity $(\bar{v})$, maximum velocity $\left(v_{\text {max }}\right)$, maximum acceleration $\left(a_{\max }\right)$, sensitivity to orientation $\left(\sigma_{\text {ori }} / \mu_{\text {ori }}\right)$, number of zero-crossings of the acceleration signal $\left(\sharp_{\text {jerks }}\right)$, number of drops $\left(\sharp_{\text {drops }}\right)$, and root mean square of the forces $\left(\sqrt{\sum F^{2}}\right)$. These values are averaged over the different movements within a trial to obtain raw parameters.

Extraction of the Scores. In order to give the physician more intuitive information, the six scores (representing different axes of mobility) were calculated using the raw parameters:

1. Velocity: indication of the displacement speed from one point to another. A low velocity could reveal muscle weakness or abnormal muscle tone.

2. Acceleration: indication of the capacity to reach a displacement speed in a short time. A lack of force could be the cause of a weak acceleration.

3. Stability: if the patient trembles (repeated inversion of the acceleration sign), encounters difficulties to keep the buttons pressed or often collides with virtual elements, this is interpreted as a lack of stability. 
Table 1. The raw parameters and their weights to compute the scores

\begin{tabular}{l|cccccccccc} 
Weights & $t_{\text {reac }}$ & $t_{\text {appro }}$ & $\bar{v}$ & $v_{\max }$ & $a_{\max }$ & $\sqrt{\sum F^{2}}$ & $\sharp$ drops & $\sharp$ jerks & $\sigma_{\text {ori }} / \mu_{\text {ori }}$ \\
\hline Velocity & 0 & 0 & 0.5 & 0.5 & 0 & 0 & 0 & 0 & 0 \\
Acceleration & 0 & 0 & 0 & 0 & 1 & 0 & 0 & 0 & 0 \\
Stability & 0 & 0 & 0 & 0 & 0 & 0.2 & 0.3 & 0.5 & 0 \\
Precision & 0 & 0.7 & 0 & 0 & 0 & 0.3 & 0 & 0 & 0 \\
Flexibility & 0 & 0.5 & 0 & 0 & 0 & 0 & 0 & 0 & 0.5 \\
Reactivity & 1 & 0 & 0 & 0 & 0 & 0 & 0 & 0 & 0 \\
\hline
\end{tabular}

4. Precision: highlights the patient's ability to align his/her hand quickly and precisely with a peg or hole (without pushing too much into the walls).

5. Flexibility: the patient's ability to move his/her wrist in order to access complex orientations with the stylus; this is revealed, on one hand, by the differences between the approach times of the pegs depending on their orientation and, on the other hand, by the approach speed of the pegs in general.

6. Reactivity: measure based on the time required to initiate a movement once a peg is grasped or inserted.

Each raw parameter is rated between 0 (very bad) and 100 (excellent) using an arctan function and two baselines. The baseline for a good result is set by the mean of all healthy subjects (corresponding to a score of 80) and the one for a bad result is arbitrarily set (corresponding to 20). Then the scores are calculated with weighted averages as shown in Table1.

\section{Results and Discussion}

Comprehensibility. Healthy subjects who participated in this study quickly understood the principle and carried out the virtual test with a relative ease. The main issue that was encountered is the necessity to precisely superimpose the cursor to the pegs. Indeed, there is no force feedback between the cursor and the peg and the only feedback is visual, which was not sufficient for some users. A learning effect was visible even after the training sessions (for each subject, last session is around $12 \%$ faster than the first one). Another issue was due to the limited workspace of the haptic device, which prevented some orientations of the stylus. Some pegs were thus only accessible with one specific orientation of the stylus, resulting in additional time to grab the virtual peg. This was countered by taking into account these limitations when setting the initial orientation of the virtual pegs.

General Trends. The recorded trajectories and forces clearly showed a repeatable pattern that was observed for every peg and each user. First, the cursor followed a coarse displacement to approach a peg, followed by a fine and slow move to precisely align with the peg. Subjects then performed another coarse movement up to a hole followed by a slow movement to insert the peg (Fig. 3). 


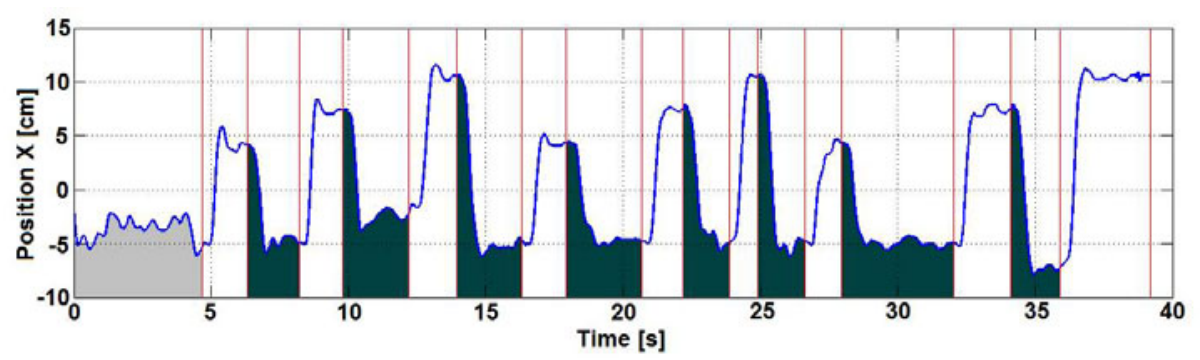

Fig. 3. X position in function of time in a typical session. Light grey: start phase; white: from peg to hole; and dark grey: from hole to peg.

Comparison between Real and Virtual Tests. When the total completion times of the real and virtual tests are compared, it appears that the virtual one is around three times longer than the real one. This is essentially due to the time required to align the cursor with the pegs. While in the real task the fingers allow grasping pegs in different orientations without turning the hand, this is not possible in the virtual task where the stylus needs to be aligned with the peg. Nevertheless, there is a good correlation between average completion times for the real and virtual test $(\mathrm{R}=0.77)$. This suggest that the virtual test gives results that are similar to the real NHPT, and validates its use as a tool to assess dexterity. In addition to the completion time, the virtual test offers the possibility of extracting additional values to quantitatively and objectively assess fine hand function.

Effect of Hand Dominance on Virtual Tests. An ANOVA was performed on total completion times depending on used hand and hand dominance (Table 2) and showed

Table 2. Mean and standard deviation of the completion time for the real and the virtual NHPT (a) and effect of hand dominance and used hand on the virtual test(b)

(a)

\begin{tabular}{l|lrcc} 
Test & Hand & $\mathrm{N}$ & Mean [s] & SD [s] \\
\hline Real & Dominant hand & 30 & 12.01 & 1.39 \\
& Non-dominant hand 30 & 12.69 & 1.29 \\
\hline Virtual & Dominant hand & 30 & 32.69 & 9.6 \\
& Non-dominant hand 30 & 35.29 & 11.43 \\
\hline
\end{tabular}

(b)

\begin{tabular}{l|lccc} 
Dominance & Tested hand N & Mean [s] & SD [s] \\
\hline \multirow{2}{*}{ Right Hand } & Right & 24 & 33.68 & 10.45 \\
& Left & 24 & 36.54 & 12.45 \\
\hline \multirow{2}{*}{ Left Hand } & Right & 6 & 30.3 & 1.63 \\
& Left & 6 & 28.74 & 2.16 \\
\hline
\end{tabular}


no significant differences between dominant and non-dominant hands $(\mathrm{F}(1 ; 58)=0.88$, $\mathrm{p}$ $\gg 0.05)$ and between left- and right-handed subjects $(F(1 ; 58)=2.68, \mathrm{p}>0.05)$.

Tests with Specific Behaviors. In order to evaluate the ability of the program to discriminate hand function according to the six chosen axes of mobility, a control experiment was conducted where specific characteristic impairments of hand mobility were simulated: tremor, with high frequency arm movements; weakness, by adding an additional mass to the hand; inaccurate, requiring excessive time to align to pegs and holes; rough, exerting high forces against the virtual board; slow, moving at low velocity. Results were compared to an optimal performance session by computing scores based on the raw parameters. Figure 4 presents the visual representation of scores, and demonstrates that the selected parameters could be used to detect specific impairments. For instance, the inaccurate behavior gave a low score on the precision axis, and the slow behavior resulted in a low velocity score and a low acceleration score.

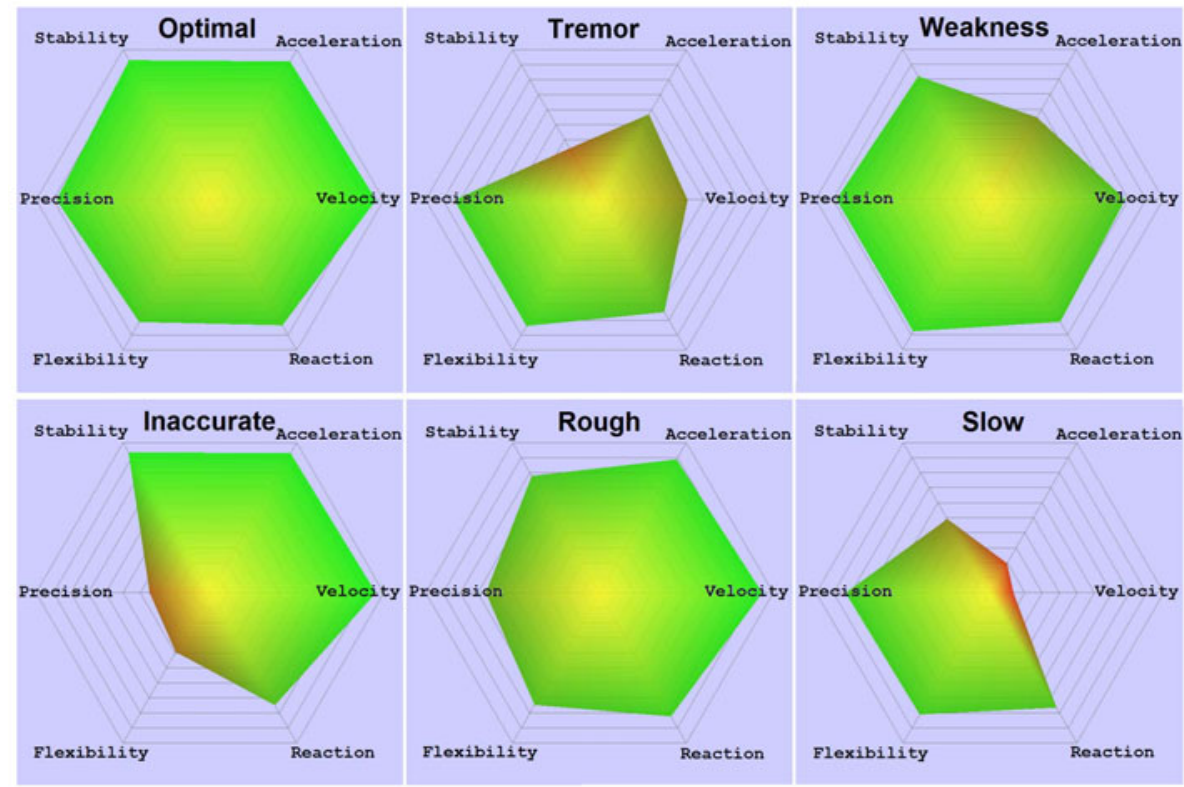

Fig. 4. Scores representing different simulated behaviors

\section{Conclusion}

A virtual counterpart of the established NHPT has been designed, implemented on a low-cost PHANTOM Omni device, and validated as a powerful VR tool for the assessment of dexterity and related impairments. This virtual test is easy to understand and perform. The main features of the hand movement can easily be detected and are found in every trial among all healthy subjects, which allows to analyze the raw data with a generalized algorithm and to fix baselines representing a healthy user's scores. In addition, the axes of mobility defined in this study reflect the fine hand motor functions of 
the user and successfully reveal the simulated impairments. As the current test group only involved subjects aged less than 38 years, it is possible that the results might not be generalized for subjects that are not used to virtual reality environments. Future work will focus on establishing a baseline for different age groups as well as with subjects suffering from impaired hand function, e.g., after stroke.

Acknowledgments. Olivier Lambercy and Roger Gassert are supported by the NCCR Neural Plasticity and Repair, Swiss National Science Foundation.

\section{References}

1. Amirabdollahian, F., Gomes, G.T., Johnson, G.R.: The Peg-in-Hole: A VR-Based Haptic Assessment for Quantifying Upper Limb Performance and Skills. In: IEEE 9th International Conference on Rehabilitation Robotics (2005)

2. Choi, S., Tan, H.Z.: Discrimination of Virtual Haptic Textures Rendered with Different Update Rates. In: Proc. of the 1st Joint Eurohaptics Conference and Symp. on Haptic Interfaces for Virtual Environment and Teleoperator Systems (2005)

3. Feys, P., et al.: Arm training in Multiple Sclerosis using Phantom: clinical relevance of robotic outcome measures. In: IEEE 11th Int. Conf. on Rehabilitation Robotics (2009)

4. Gomes, G.T., Amirabdollahian, F., Johnson, G.R.: Quantifying upper limb motor control: the Peg in Hole test. In: ISB XXth Congress - ASB 29th Annual Meeting (2005)

5. Hannaford, B., Wood, L., McAffee, D.A., Zak, H.: Performance Evaluation of a Six-Axis Generalized Force-Reflecting Teleoperator. IEEE Transactions on Systems, Man, and Cybernetics 21(3) (1991)

6. Mathiowetz, V., Weber, K., Kashman, N., Volland, G.: Adult norms for the Nine Hole Peg Test of finger dexterity. Occup. Ther. J. of R 5(1), 24-38 (1985)

7. Milner, T.E.: A model for the generation of movements requiring endpoint precision. Neuroscience 49(2), 487-496 (1992)

8. Oxford Grice, K., Vogel, K., Le, V., Mitchell, A., Muniz, S., Vollmer, M.: Adult norms for a commercially available Nine Hole Peg Test for finger dexterity. Am. J. Occup. Ther. 57(5), 570-573 (2003)

9. Unger, B.J., Nicolaidis, A., Berkelman, P.J., Thompson, A., Klatzky, R.L., Hollis, R.L.: Comparison of 3-D haptic peg-in-hole tasks in real and virtual environments. In: IEEE/RSJ, IROS, pp. 1751-1756 (2001)

10. Xydas, E.G., Louca, L.S.: Design and Development of a Haptic Peg-Board Exercise for the Rehabilitation of People with Multiple Sclerosis. In: IEEE 10th International Conference on Rehabilitation Robotics (2007) 